\title{
IL-6 induces regionally selective spinal cord injury in patients with the neuroinflammatory disorder transverse myelitis
}

\author{
Adam I. Kaplin, ${ }^{1}$ Deepa M. Deshpande, ${ }^{2}$ Erick Scott, ${ }^{3}$ Chitra Krishnan, ${ }^{2}$ \\ Jessica S. Carmen, ${ }^{3}$ Irina Shats, ${ }^{3}$ Tara Martinez, ${ }^{3}$ Jennifer Drummond, ${ }^{2}$ Sonny Dike, ${ }^{2}$ \\ Mikhail Pletnikov, ${ }^{1}$ Sanjay C. Keswani, ${ }^{2}$ Timothy H. Moran, ${ }^{1}$ Carlos A. Pardo, ${ }^{2,4}$ \\ Peter A. Calabresi, ${ }^{2}$ and Douglas A. Kerr ${ }^{2,3}$
}

\begin{abstract}
1Department of Psychiatry and Behavioral Sciences, ${ }^{2}$ Department of Neurology, ${ }^{3}$ Department of Molecular Microbiology and Immunology, Bloomberg School of Public Health, and 4Department of Pathology, Johns Hopkins University School of Medicine, Baltimore, Maryland, USA.
\end{abstract}

\begin{abstract}
Transverse myelitis (TM) is an immune-mediated spinal cord disorder associated with inflammation, demyelination, and axonal damage. We investigated the soluble immune derangements present in TM patients and found that IL-6 levels were selectively and dramatically elevated in the cerebrospinal fluid and directly correlated with markers of tissue injury and sustained clinical disability. IL- 6 was necessary and sufficient to mediate cellular injury in spinal cord organotypic tissue culture sections through activation of the JAK/STAT pathway, resulting in increased activity of iNOS and poly(ADP-ribose) polymerase (PARP). Rats intrathecally infused with IL-6 developed progressive weakness and spinal cord inflammation, demyelination, and axonal damage, which were blocked by PARP inhibition. Addition of IL- 6 to brain organotypic cultures or into the cerebral ventricles of adult rats did not activate the JAK/STAT pathway, which is potentially due to increased expression of soluble IL- 6 receptor in the brain relative to the spinal cord that may antagonize IL- 6 signaling in this context. The spatially distinct responses to IL-6 may underlie regional vulnerability of different parts of the CNS to inflammatory injury. The elucidation of this pathway identifies specific therapeutic targets in the management of CNS autoimmune conditions.
\end{abstract}

\section{Introduction}

Transverse myelitis (TM) is a focal inflammatory disorder of the spinal cord and exists on a spectrum of neuroinflammatory conditions characterized by abrupt neurologic deficits associated with inflammation, demyelination, and axonal damage (1). TM can exist as part of a multifocal CNS disease (e.g., MS), a multi-system disease (e.g., systemic lupus erythematosus), or as an isolated idiopathic entity. We investigated the diffusible immune derangements present in the spinal fluid of idiopathic TM patients and report our progress elucidating the role of IL- 6 in the pathogenesis of this disease.

IL-6 is a glycoprotein cytokine that mediates signal transduction between immune cells, induces acute-phase protein synthesis, and controls growth and differentiation of cells of the immune and hematopoietic systems (2). IL-6 likely is a trophic factor that, under some circumstances, supports neuronal and glial differentiation and survival (2). Introduction of members of the IL-6 superfamily, including IL-6 itself, in some systems has been shown to ameliorate demyelination, perhaps by providing trophic support for oligodendrocytes (3).

In addition to these potentially beneficial effects of IL-6, there has been a growing appreciation of the destructive potential of

Nonstandard abbreviations used: 4-ANI, 4-amino-1, 8-napthalimide; APP, amyloid precursor protein; CSF, cerebrospinal fluid; EAE, experimental allergic encephalomyelitis; EDSS, expanded disability status scale; GFAP, glial fibrillary acidic protein; IL-6R, IL-6 receptor; NT, nitrotyrosine; $\mathrm{ONOO}^{-}$, peroxynitrite anion; PARP, poly(ADP-ribose) polymerase; RIP, receptor interacting protein; sIL-6R, soluble IL-6R; TM, transverse myelitis.

Conflict of interest: The authors have declared that no conflict of interest exists.

Citation for this article: J. Clin. Invest. 115:2731-2741 (2005).

doi:10.1172/JCI25141. elevated levels of IL-6 in the CNS (2). IL-6 levels in the adult CNS are usually low or undetectable under baseline conditions, but increase dramatically in response to injury, inflammation, and CNS disease. TNF- $\alpha$, IL- $1 \beta$, and neurotransmitters are among the most important stimulators of IL- 6 production from astrocytes and microglia within the CNS (4). There is indirect evidence that elevated IL- 6 potentiates neural injury in Alzheimer disease, Parkinson disease, HIV encephalopathy, MS, depression, and cognitive impairment $(4,5)$. Transgenic mice that overexpress IL-6 within astrocytes exhibit ataxia, seizures, and hind limb paralysis and have extensive neurodegeneration (6). Conversely, IL-6 KO mice are resistant to experimental allergic encephalomyelitis (EAE) (7), and IL-6-neutralizing antibodies reduce EAE in wild-type mice (8), suggesting a role for IL-6 in mediating autoimmune demyelination. Neutralization of CNS IL- 6 attenuates traumatic spinal cord injury in rats and is associated with reduced iNOS activity (9). As has been seen in other tissues (10), IL-6 within the CNS may stimulate iNOS expression, resulting in the production of $\mathrm{NO}$ and leading to free radical-induced tissue injury (10).

IL-6 produces its effects by binding to IL-6 receptors (IL-6Rs), which form complexes with gp130. Once formed, the IL-6/IL-6R/ gp130 complex stimulates the following 2 main signal transduction cascades that lead to activation of a number of transcription factors responsible for IL-6-mediated effects: JAK/STAT and Ras/MEK/ MAPK (11). IL-6-induced activation of the JAK2/STAT3 signaling pathway in cardiac myocytes results in activation of iNOS with subsequent NO production and decreased cardiac contractility (10).

iNOS is normally expressed either minimally or not at all in the CNS, but in pathological conditions, iNOS levels can increase dramatically in glial cells or influxing macrophages in response 

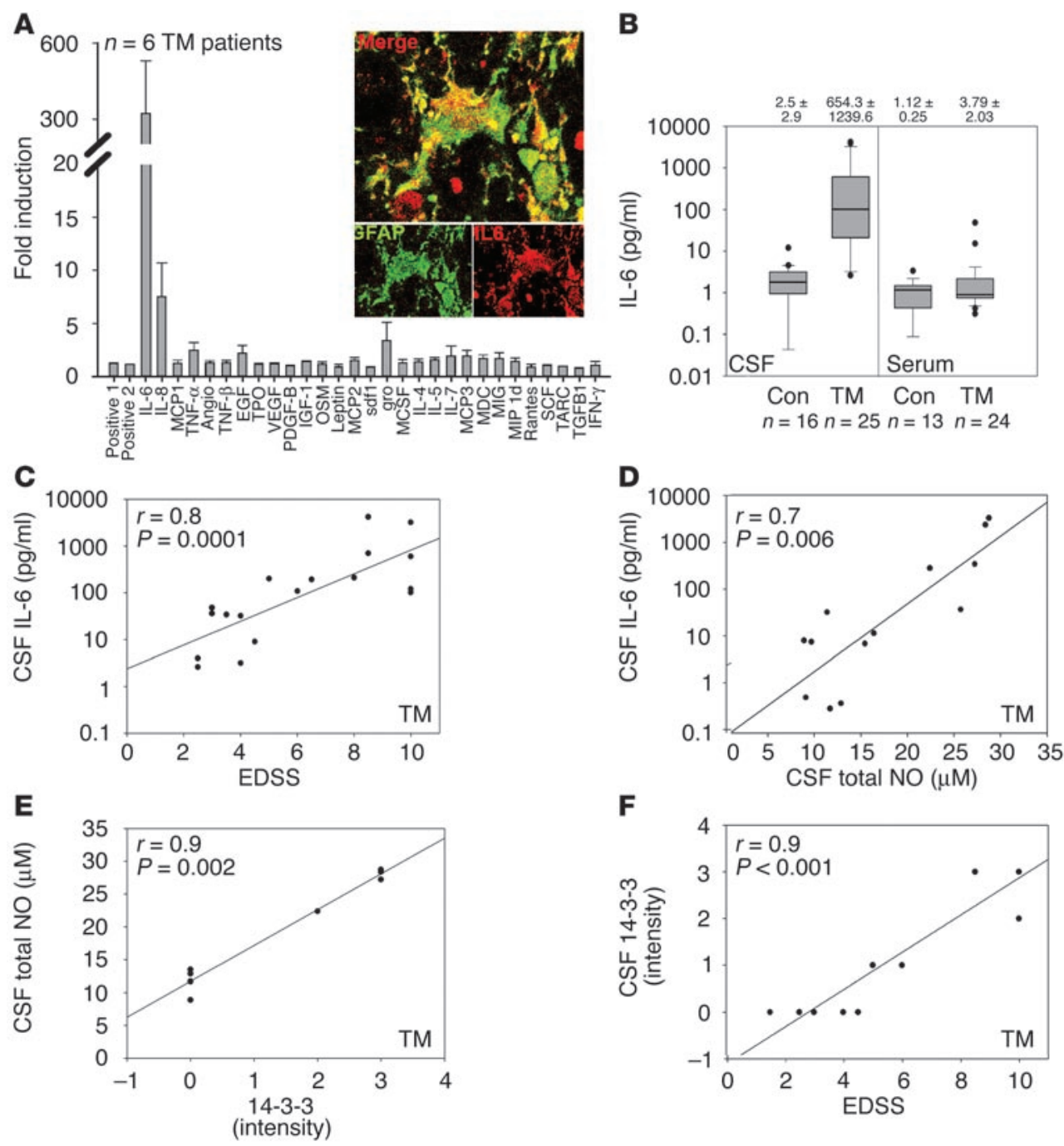

Figure 1

IL-6 is selectively upregulated in the CSF of TM patients and correlates with long-term disability. (A) Cytokine array was used to profile 42 inflammatory proteins in the CSF of 6 TM and 8 control patients. The mean value of each cytokine was defined for the control group, and fold induction was calculated for each TM patient. Inset: IL-6 immunohistochemistry was performed on the cervical spinal cord of a TM patient who died of respiratory failure; IL-6 expression colocalized with GFAP-positive astrocytes. Magnification, $\times 60$. (B) Quantitative IL-6 levels in the CSF and serum of control (Con) and TM patients were determined by ELISA. Box plots represent the interquartile range, and the outliers shown are outside the fifth and ninety-fifth percentiles. Mean \pm SEM for each group is indicated above each box. (C) Among TM patients, acute CSF IL-6 levels strongly correlated with sustained disability (as measured by EDSS). (D) CSF IL-6 levels strongly correlate with total NO metabolites during the acute phase of TM. (E) Total NO correlates with 14-3-3, a neuronal injury marker in TM patients. (F) Levels of 14-3-3 strongly correlate with sustained disability in TM patients. For panels $\mathbf{C}-\mathbf{F}$, correlation coefficients and statistical significance are shown. Intensity, chemiluminescent signal intensity.

to injury or inflammation (12). Compared with constitutive forms of NOS (neuronal and constitutive NOS), iNOS generates significantly greater, sustained amounts of NO (picomolar vs. micromolar levels, respectively) (13). Expression of iNOS causes delayed neurotoxicity in mixed neuronal-glial cortical cultures (14), and NO is directly cytotoxic to oligodendrocytes in culture (15). iNOS immunoreactivity is elevated in brains of MS patients relative to controls (16). Increased expression of iNOS mRNA is also found in EAE, and inhibition of iNOS protects animals from
EAE (17). The destructive influence of iNOS following spinal cord trauma has been demonstrated in iNOS KO mice, which had better functional outcomes from compression injury than wild-type controls (18).

Much of NO-mediated pathogenicity depends on the formation of secondary intermediates such as the peroxynitrite anion $\left(\mathrm{ONOO}^{-}\right)$(19). ONOO- triggers DNA single-strand breakage that activates the DNA-repair enzyme poly(ADP-ribose) polymerase (PARP) (20). PARP catalyzes the transfer of ADP-ribose from its substrate $\mathrm{NAD}^{+}$to various proteins, including histones $(21,22)$. Excessive activation of PARP in response to extensive DNA damage has been associated with the depletion of $\mathrm{NAD}^{+}$, which causes cell death through energy depletion (22). It was recently reported that $\mathrm{ONOO}^{-}$induced toxicity in spinal cord neurons is associated with DNA strand breakage and prevented by PARP inhibition (23).

In the current study, we found that IL- 6 was elevated in the cerebrospinal fluid (CSF) of TM patients at the time of their acute clinical presentation and correlated with the patients' eventual long-term disability. By utilizing organotypic cultures and developing an animal model of TM, we demonstrate that IL- 6 is both necessary and sufficient to mediate the kind of spinal cord injury found in patients with TM. NO production is necessary to achieve this tissue damage. We provide evidence that the targets of this IL-6-mediated injury are oligodendrocytes and axons, which result in demyelination and axonal injury. To our knowledge, this work provides evidence for the first time that a single signaling protein is the central mediator of tissue injury in an autoimmune CNS disease. We also describe the signaling pathway of IL-6-mediated spinal cord neural injury from JAK/STAT activation to iNOS expression to PARP activation and cell death. The regional specificity of the cytotoxicity of this IL-6 signaling pathway, which involves the spinal cord but not the brain, provides the beginning of an explanation for the selective involvement of different regions of the CNS in autoimmune spectrum diseases. 
A
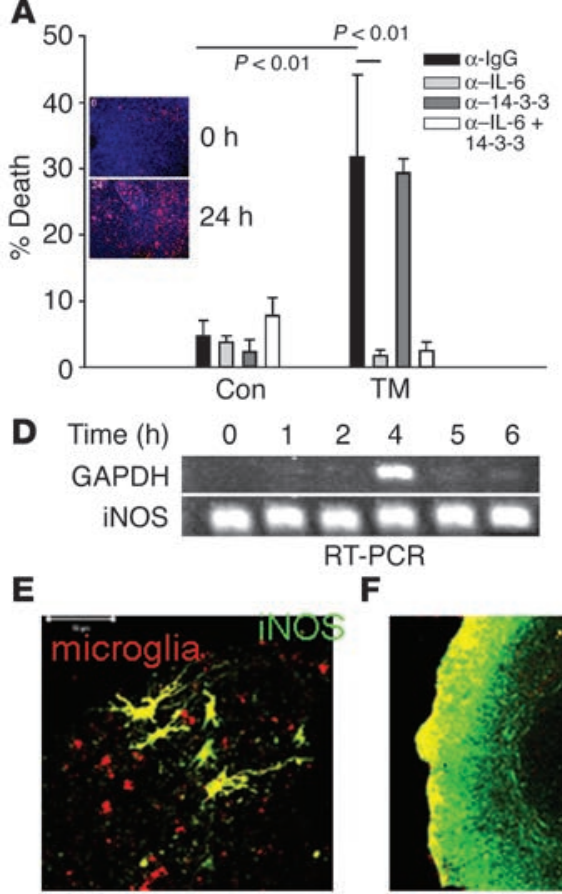

B
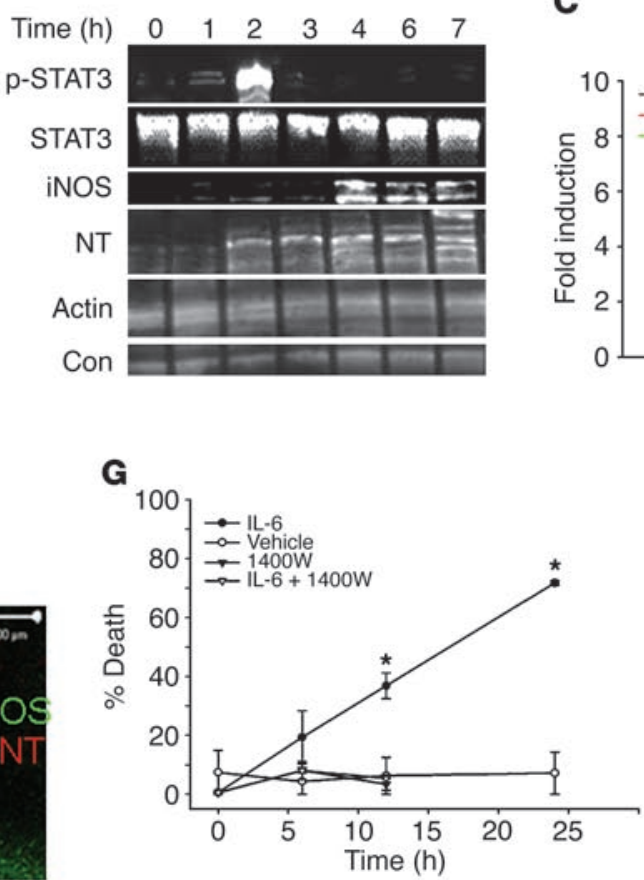

C

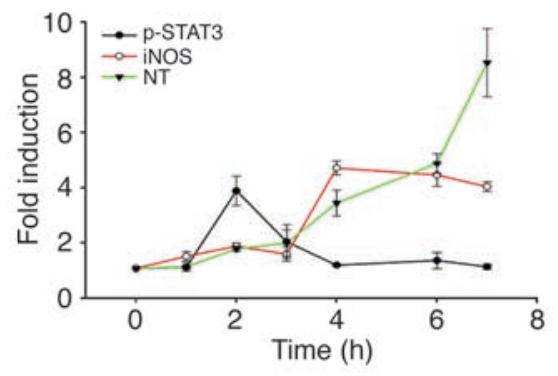

$\mathbf{H}_{100}$

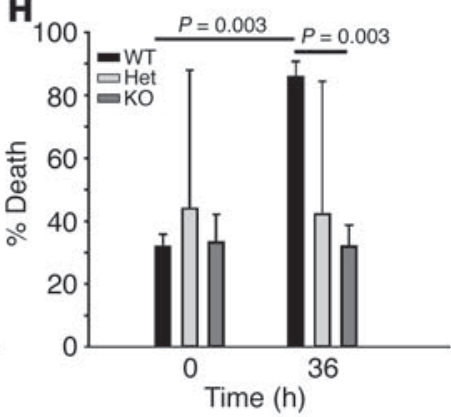

Figure 2

IL-6 is necessary and sufficient to induce injury of oligodendrocytes and axons in spinal cord organotypic cultures by generating NO. (A) CSF $(100 \mu \mathrm{l})$ from a TM or control patient was added to culture media of spinal cord organotypic cultures, and cellular injury was assessed by ethidium homodimer uptake with Hoechst counterstain (inset). IL-6 was immunodepleted by preincubating TM CSF with an IL- 6 antibody and clearing the IL-6 antibody complex with protein A sepharose. Magnification, $\times 20$. (B) Tissue lysates from spinal cord organotypics were generated at various times after the administration of IL-6 and subjected to SDS-PAGE followed by immunoblot analysis. (C) Quantification of the data shown in B by chemiluminescent signal intensity of 3 independent experiments. (D) RT-PCR analysis of RNA derived from spinal cord organotypic cultures at the indicated times after addition of IL-6 at $2,000 \mathrm{pg} / \mathrm{ml}$. (E) Dual-color confocal microscopy was carried out with spinal cord organotypic cultures treated with IL-6 for 24 hours. Microglia were identified by incubating live cultures with Dil-Ac-LDL, which is endocytosed by phagocytosing cells. After fixation, iNOS immunohistochemistry was carried out, revealing the expression of iNOS within microglia. Scale bar: $50 \mu \mathrm{m}$. (F) NT and iNOS preferentially accumulated within the exterior white matter of spinal cord organotypic cultures. Scale bar: $200 \mu \mathrm{m}$. (G) Addition of IL-6 to a final concentration of $2,000 \mathrm{pg} / \mathrm{ml}$ to spinal cord organotypic cultures in the presence or absence of the iNOS inhibitor $1400 \mathrm{~W}$. ${ }^{*} P<0.05$. (H) WT and iNOS-heterozygous and -KO spinal cord organotypic cultures were exposed to IL-6 and assessed for cellular death.

\section{Results}

IL-6 levels are selectively and dramatically elevated in the CSF of TM patients. A cytokine antibody array was used to simultaneously profile 42 inflammatory proteins in the CSF of 6 TM patients who had not been started on immunomodulatory therapy prior to CSF sampling and 8 control patients (hydrocephalus, aseptic meningitis, spinal cord infarct, and spinal cord tumors; $n=2$ per group). Relative to controls, arrays from the TM patients showed an approximately 300 -fold mean induction of IL-6 $(P<0.05)$, while all other cytokines were altered less than 10 -fold ( $P=$ NS; Figure $1 \mathrm{~A})$. Two TM patients died of respiratory failure, and we performed immunohistochemical staining of the spinal cord at autopsy in order to define the source(s) of IL-6. In both cases, we found that the predominant source of IL- 6 secretion was from astrocytes in and around the area of inflammation within the spinal cord (Figure 1A, inset), while microglial cells and influxing immune cells exhibited less robust IL- 6 staining (data not shown). To ascertain the absolute levels of IL- 6 in the serum and CSF of TM patients, we carried out quantitative ELISA in a larger series of TM $(n=25)$ and control patients $(n=16$; Figure 1B). Mean CSF IL-6 levels in patients with acute TM were 262-fold higher than in control patients (TM, 654.3 $\pm 247.9 \mathrm{pg} / \mathrm{ml}$, range 0-4209 pg/ml; control, $2.5 \pm 0.72 \mathrm{pg} / \mathrm{ml}$ ).
Mean serum IL-6 levels in TM and control patients were not statistically significantly different and were markedly lower than those seen in the CSF, suggesting that the IL- 6 measured in the CSF was generated within the CNS. Further details on the clinical features of the TM and control patients used in this study are provided in Supplemental Table 1 (supplemental material available online with this article; doi:10.1172/JCI25141DS1).

IL-6 levels correlate with total NO production and tissue injury in the spinal cord. We found a strong correlation between CSF IL- 6 obtained at the time of acute clinical evaluation and long-term disability (as assessed by an expanded disability status scale [EDSS] at 6-month follow-up; Figure 1C). We found a significant correlation between acute CSF IL-6 levels and acute CSF total NO levels (Figure 1D) in TM patients and that total CSF NO correlates with 14-3-3, a previously reported marker of acute neuronal injury in TM patients (24) (Figure 1E). Further, we found that CSF 14-3-3 levels correlated with long-term disability (Figure 1F). These data are consistent with a direct role of NO in mediating spinal cord injury.

IL-6 is necessary and sufficient to cause spinal cord cellular injury by activating the JAK/STAT pathway, which results in increased iNOS activity. To test whether IL- 6 is simply correlated with or is causative of cellular injury in the spinal cord, we carried out studies using rat 

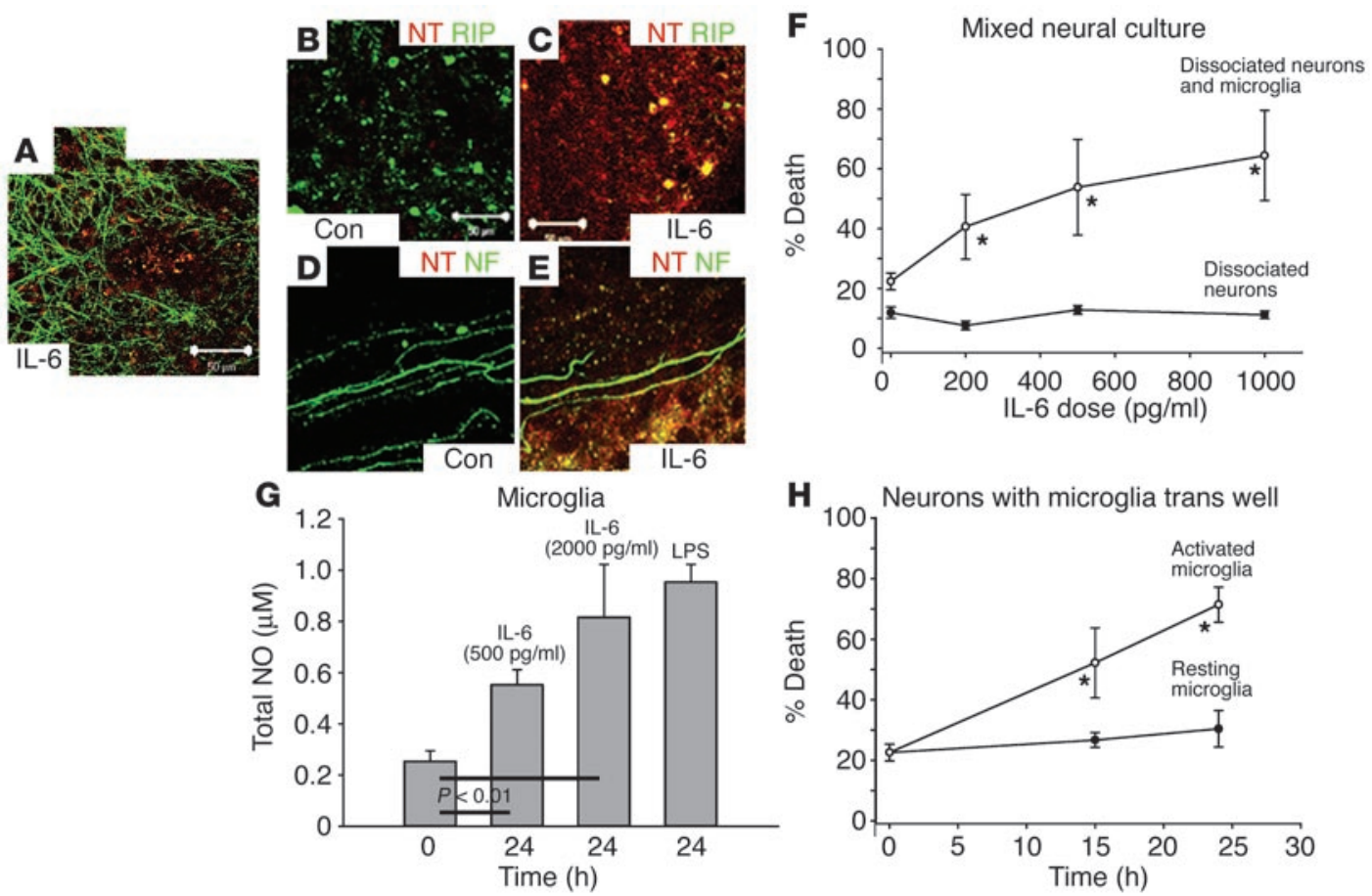

Figure 3

Cellular participants in IL-6-induced injury. (A-E) We performed dual-color confocal microscopy using an antibody to NT as a marker of NO excess and the cell-specific markers GFAP, RIP, and neurofilament (NF). (F) Dissociated rat spinal neuron cultures were exposed to IL-6 either alone or 2 days after plating $10^{4}$ purified microglial cells in coculture with the dissociated neurons. (G) Purified, cultured microglial cells were exposed to either 500 or $2,000 \mathrm{pg} / \mathrm{ml}$ of IL-6. Twenty-four hours later, supernatants were harvested and examined for total nitrates produced by the culture cells. LPS, lipopolysaccharide. (H) Microglia were plated in a trans well, exposed to IL-6 for 2 hours, and then washed. They were then transferred to 24-well dishes with plated, dissociated neurons, and the neurons were assessed for cellular death up to 24 hours later. ${ }^{*} P<0.05$.

organotypic culture spinal cord sections. We added CSF from a TM patient (with IL-6 of $1,997 \mathrm{pg} / \mathrm{ml}$ ) or a control patient onto spinal cord organotypic culture sections and evaluated cell death by determining the percentage of cells that took up the red, vital dye propidium iodide. We found that CSF from the TM patient induced death of spinal cord cells $(33 \% \pm 12 \%)$, while CSF from a control patient with hydrocephalus did not $(4 \% \pm 2.4 \%, P<0.01$; Figure $2 \mathrm{~A}$, black bars and photomicrograph inset). Immunodepletion of IL- 6 from the CSF prior to application (decrease in IL-6 to $2 \mathrm{pg} / \mathrm{ml}$; data not shown) abrogated the ability of the CSF to induce death, suggesting that IL- 6 was necessary for this death $(P<0.01$; Figure $2 \mathrm{~A})$. Immunodepletion of IgG or a control protein (14-3-3) did not abrogate the killing activity of TM CSF. We found that IL- 6 at a dose found in the CSF of TM patients $(2,000 \mathrm{pg} / \mathrm{ml})$ triggered a dramatic, transient phosphorylation of STAT3 at Tyr705, peaking 2 hours after the application to the culture medium of spinal cord organotypic cultures (total STAT levels were unchanged; Figure 2, B and C). We also saw a peak of iNOS expression at 4 hours after IL- 6 administration. Nitrosylation of tyrosine residues (nitrotyrosine [NT]), a marker of NO excess (25), continued to increase at 7 hours after IL- 6 administration. Actin and loading controls did not show any change over the examined period. Quantitative determination of phosphorylated STAT3, iNOS, and NT intensity of several representative immunoblots revealed a nearly 4-fold increase of phosphorylated STAT3 at 2-3 hours, a nearly 4.5 -fold increase of iNOS at 4 hours, and a progressive increase in NT immunoreactivity (greater than 8-fold) at
7 hours after IL-6 administration (Figure 2C). RT-PCR analysis of spinal cord organotypic cultures confirmed a marked upregulation of iNOS RNA at 4 hours after IL-6 administration (Figure 2D), demonstrating that the upregulation of iNOS occurs at the level of transcription. Immunofluorescent examination of spinal cord organotypic cultures revealed a dramatic upregulation of iNOS within microglial cells (defined by the ability to phagocytose fluorescent acetylated low-density lipoprotein [DiI-Ac-LDL]; Figure 2E) and the accumulation of both iNOS and NT immunoreactivity, especially within the peripheral white matter of the spinal cord (Figure 2F), in response to IL-6 administration.

Next, we investigated the consequences of iNOS induction in spinal cord organotypic cultures and found that iNOS is critical for IL-6-induced death (Figure 2G). We administered IL-6 to the culture medium and found that the progressive cellular injury that occurs is completely blocked by $1400 \mathrm{~W}$ (Figure $2 \mathrm{G}$ ). Similarly, mouse organotypic spinal cord cultures from either iNOS-heterozygous or -KO mice were resistant to IL-6-induced death (Figure 2H). This suggests that IL-6 induces activation of STAT3, resulting in the upregulation of iNOS and cellular injury within the spinal cord.

Targets of IL-6-mediated spinal neural injury. We identified the target cells injured by IL- 6 by examining the colocalization of NT, a commonly utilized marker of cellular injury due to excess NO (26), with other cellular markers and found that NT immunoreactivity was diffusely increased within IL-6-treated tissue (Figure 3, A, C, and E). Additionally, while astrocytes (defined by glial fibrillary 

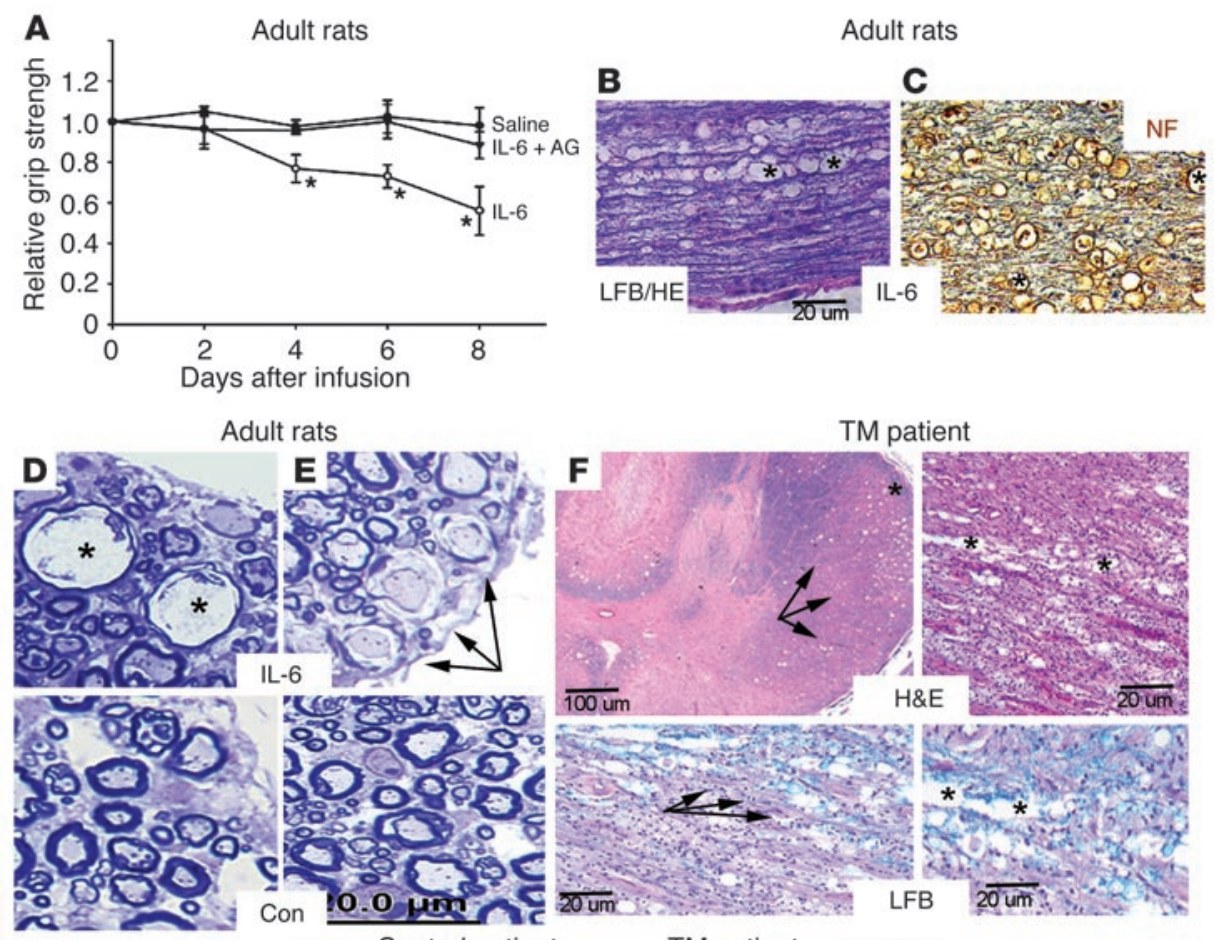

Adult rats
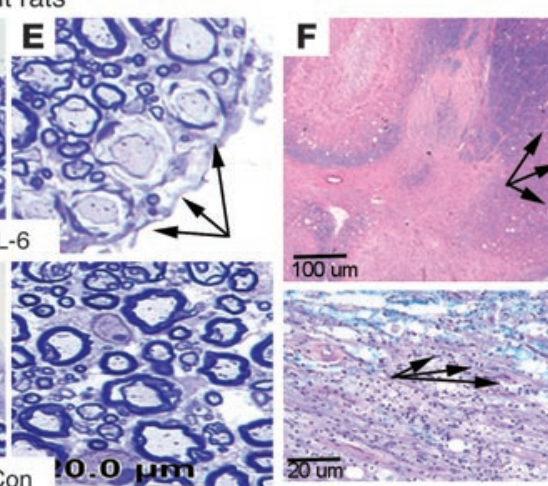

TM patient

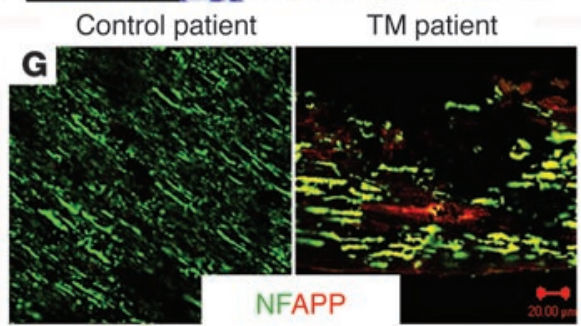

Figure 4

IL-6 induces weakness with axonal degeneration and loss of myelin when infused into the spinal subarachnoid space of adult rats. (A) We infused IL-6 via a subarachnoid spinal catheter into adult rats over a 7-day period. Control animals received saline through the spinal catheter, while another cohort of animals received intrathecal IL-6 and were also given the iNOS inhibitor aminoguanidine (AG) intraperitoneally for the length of the experiment beginning at day 0 . ${ }^{*} P<0.05$. (B) Pathologic specimens from IL-6-infused rat spinal cords exhibited reduced myelin staining and white matter vacuolation (asterisks). Scale bar: 20 um. (C) White matter vacuoles (asterisks) were strongly NF positive, confirming the presence of axonal degeneration. Scale bar: $20 \mu \mathrm{m}$. (D and E) Plastic sections $(1 \mu \mathrm{M})$ from IL-6-infused rat spinal cords revealed 2 pathologic features, seen principally in superficial white matter regions: (D) swollen axons with intact myelin (asterisks), consistent with axonal degeneration, and (E) demyelinated axons (arrows). Scale bar: $10 \mu \mathrm{m}$ (D and E). (F) These pathologic features could also be seen in autopsy material from a patient with severe TM and high CSF IL-6 levels (1,997 pg/ml). Regions of demyelination (arrows) were seen throughout the cervical spinal cord both with H\&E staining and with luxol fast blue (LFB), and there were areas of vacuolation within the white matter (asterisks), consistent with axonal degeneration. (G) Immunohistochemical analysis of the autopsy material also revealed axonal dysfunction as defined by disruption of NF staining and accumulation of APP, a marker of disrupted axonal transport.

acidic protein [GFAP] immunoreactivity) did not accumulate NT in IL-6-treated cultures (Figure 3A), oligodendrocytes (defined by receptor interacting protein [RIP] immunoreactivity; Figure 3, $B$ and $C$ ) and axons (defined by neurofilament immunoreactivity; Figure 3, D and E) did and were therefore preferentially susceptible to IL-6-induced injury. When we generated dissociated cultures of pure spinal neurons, we found that IL- 6 did not induce cellular death at any of the studied time points or doses (Figure 3F). When we added microglia to the mixed neural culture either in cis (Figure 3F) or in trans (Figure 3H), we restored the ability of IL- 6 to induce neural injury, confirming that microglial cells are critical in this pathway. Mixed cultures of neurons and astrocytes were also refractory to IL-6-induced cytotoxicity (data not shown). Indeed, purified microglia were induced to secrete high levels of $\mathrm{NO}$ in response to IL- 6 at 500 and $2,000 \mathrm{pg} / \mathrm{ml}$ (Figure 3G). These results suggest that IL- 6 activates microglia to produce NO, resulting in neural injury to spinal cord cultures.

Spinal cord from IL-6-infused rats and $T M$ patients exhibit demyelination and axonal degeneration. To determine whether elevation of spinal IL-6 in adult rats is sufficient to induce weakness and cellular injury, we infused IL-6 or vehicle through a spinal subarachnoid catheter. The catheter was attached to a subcutaneous osmotic minipump that infused IL-6 or vehicle at $0.5 \mu \mathrm{l} / \mathrm{h}$ for 7 days ( $24 \mathrm{ng}$ IL-6 infused per day). We found that whereas saline-infused animals showed no change in hind limb grip strength over the 8-day observation period, IL-6-infused rats became progressively weak $(n=10$ per group; Figure 4A). By the completion of the study, IL-6-infused rats had lost nearly $50 \%$ of their baseline hind limb strength. IL-6-infused rats treated with the iNOS inhibitor aminoguanidine did not exhibit weakness, suggesting that iNOS is required for spinal neural injury. Histologic analysis of the spinal cords from IL-6-infused animals revealed white matter disruption (Figure 4B, asterisks) and the accumulation of neurofilamentous material within white matter vacuoles (Figure 4C). Grey matter was largely spared (data not shown). The analysis of plastic-embedded $1-\mu \mathrm{m}$ histologic sections from IL-6-infused rats revealed both axonal degeneration, characterized by swollen, empty, myelin-encased chambers (Figure 4D, asterisks), and demyelination, characterized by intact axons with little or no surrounding myelin (Figure 4E, arrows).

This pathology in IL-6-infused rats was similar to the axonal degeneration and demyelination seen in the spinal cord of a patient with severe, fatal TM (the same patient with IL-6 levels of 1,997 pg/ml). Histologic analysis of the cervical spinal cord revealed focal areas of demyelination (Figure 4F, arrows) and axonal disruption (Figure 4F, asterisks). Pathologic analysis of this patient's spinal cord also revealed amyloid precursor protein (APP) accumulation 

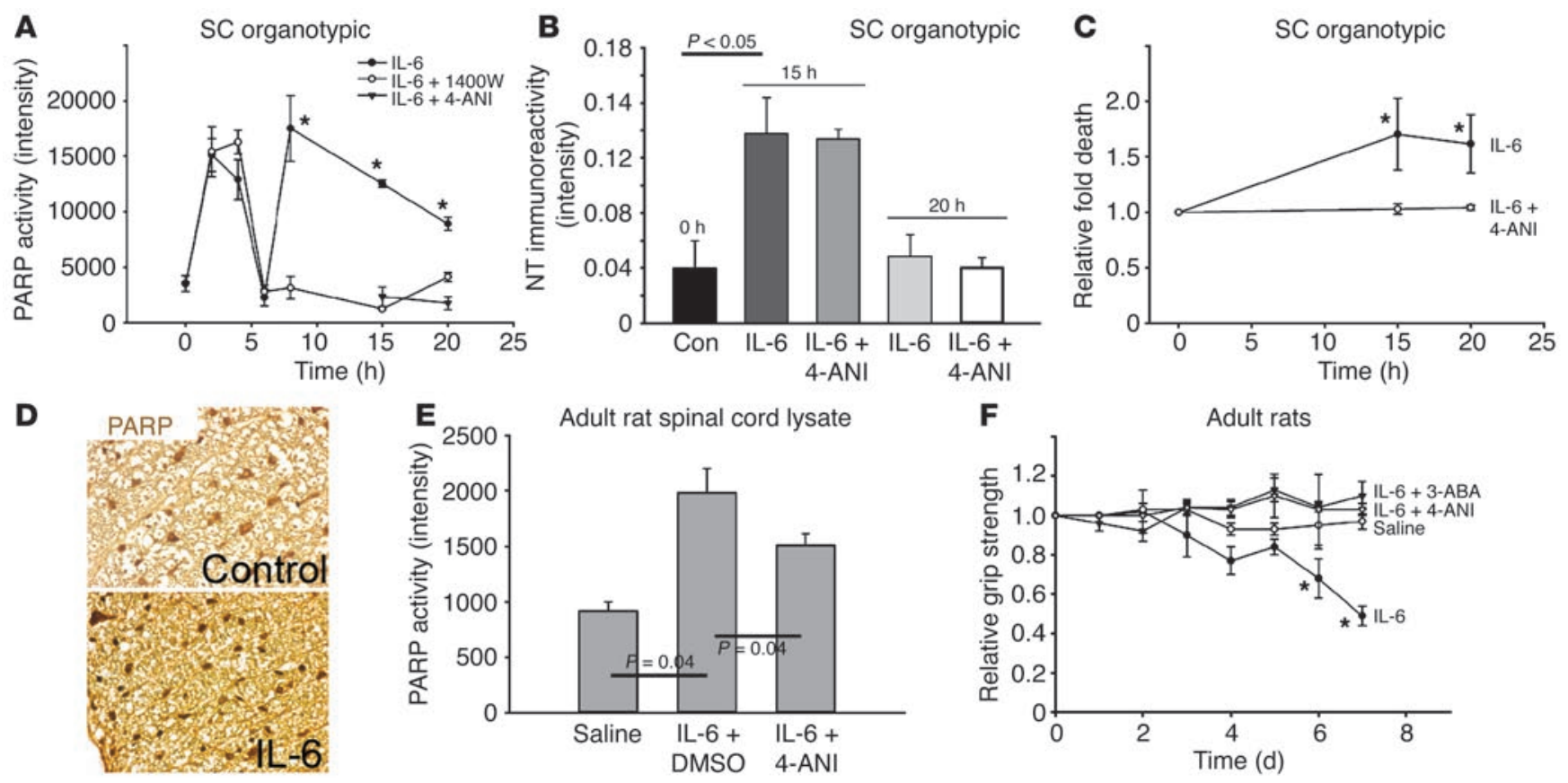

Figure 5

PARP activation is necessary for IL-6-induced toxicity. (A) PARP activity of IL-6-treated spinal cord (SC) organotypic cultures was assessed in the presence or absence of the PARP inhibitor 4-ANI or the iNOS inhibitor 1400W. (B) Spinal cord organotypic cultures were incubated with IL-6 in the presence or absence of the PARP inhibitor 4-ANI, and the amount of NT accumulation was assessed as a marker of iNOS activity. (C) Spinal cord organotypic cultures were incubated with IL-6 in the presence or absence of 4-ANI, and cell death was assessed by propidium iodide uptake. (D) PARP immunoreactivity was examined in the spinal cord of IL-6-infused rats 4 days after initiation of IL-6 infusion. Magnification, $\times 20$. (E) PARP activity was assessed in spinal cord tissue lysates generated from IL-6-infused rats, control rats, or rats both infused with IL-6 and given the PARP inhibitor 4-ANI intraperitoneally. Lysates were generated 4 days after initiation of IL-6 infusion. (F) IL-6 was infused into the spinal subarachnoid space of adult rats in the presence or absence of the PARP inhibitors 4-ANI or 3-AB given systemically. Hind limb grip strength was assessed daily for 7 days as described in Methods. ${ }^{*} P<0.05$.

within axons, a marker of axonal dysfunction, and neurofilament disruption (Figure 4G, right), whereas in a control patient, no APP accumulation was observed within axons (Figure 4G, left).

Activation of PARP is necessary for cell death. We next examined whether PARP activation contributes to IL-6-induced neural injury by using spinal cord organotypic cultures and found that there was a robust increase in PARP activity in a biphasic manner: at 2 hours and again at 8-15 hours after IL-6 administration (Figure 5A). Since it has previously been shown that the excess production of NO leads to DNA strand breaks and PARP activation, we reasoned that the second peak of PARP activity may be a consequence of iNOS activity, which is initiated $2-3$ hours following IL-6 induction. To test this hypothesis, we incubated organotypic cultures with IL-6 and the iNOS inhibitor 1400W and assessed PARP activity. As expected, the second peak of PARP activity was prevented when organotypic cultures were incubated with 1400W, confirming that iNOS activity is required. However, the first peak of PARP activity was not prevented by incubation with $1400 \mathrm{~W}$, indicating that this early peak is independent of iNOS activity. We confirmed these data by examining the activation of PARP in wild-type and iNOS KO animals (Supplemental Figure 1).

In order to define whether iNOS induction is dependent upon PARP activity, we carried out the converse experiment, in which we examined the extent of NT reactivity (a marker of iNOS activity) by ELISA in the presence and absence of PARP inhibition (Figure 5B). Here, we found that the IL-6-induced increase in NT immunoreac- tivity was not blocked by the addition of PARP inhibitor 4-amino1, 8-napthalimide (4-ANI), confirming that iNOS activation is not dependent upon PARP activation and that iNOS induction occurs upstream of PARP activation. We examined the importance of PARP in IL-6-induced spinal cellular injury by incubating spinal cord organotypic cultures with IL- 6 and either the PARP inhibitor 4-ANI or vehicle and found that the PARP inhibitor completely prevented cellular death (Figure 5C). We examined the function of PARP in adult rats infused with IL- 6 through a subarachnoid catheter draining into the low thoracic spinal cord and found that IL-6-infused rats demonstrated a dramatic increase in both the amount of nuclear PARP within the white matter (Figure 5D) and the activity of PARP (Figure 5E), suggesting that, as seen in vitro, PARP activation occurs within the spinal cord of IL-6-infused rats. Further, inhibition of PARP activity by concurrent treatment of adult rats with IL- 6 and either of 2 PARP inhibitors (4-ANI or 3-aminobenzamide [3-AB]) completely prevented IL-6-induced hind limb weakness (Figure 5F). These data suggest that PARP is activated in response to IL- 6 by at least 2 distinct pathways and that inhibition of PARP activity is protective against IL-6-induced spinal injury both in vitro and in vivo.

Regional vulnerability of the spinal cord relative to the brain. We next sought to determine whether IL-6 at concentrations seen in TM patients is universally injurious to the nervous system or selectively injurious to the spinal cord. To examine this, we incubated cortical and hippocampal organotypic culture sections with increasing 


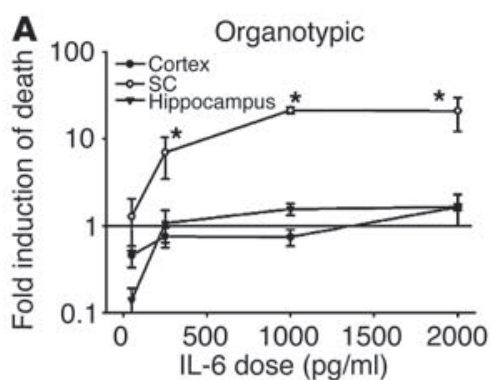

D Cortex

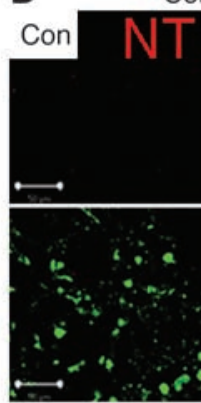

F Human tissue IL-6R

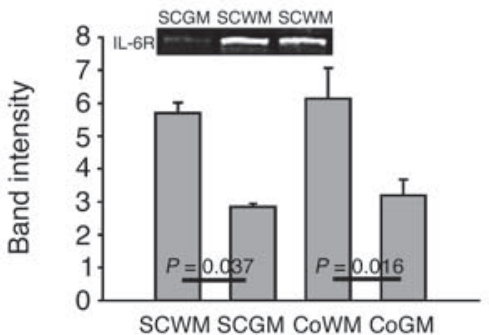

B

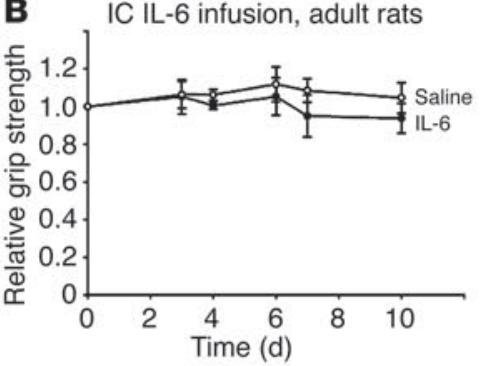

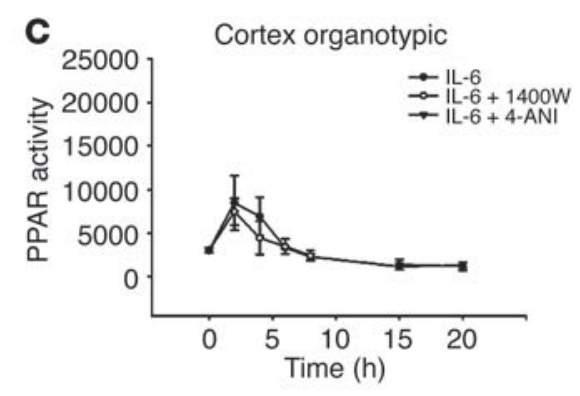

SC

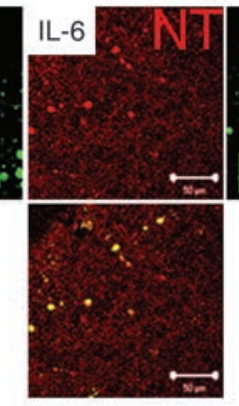

G

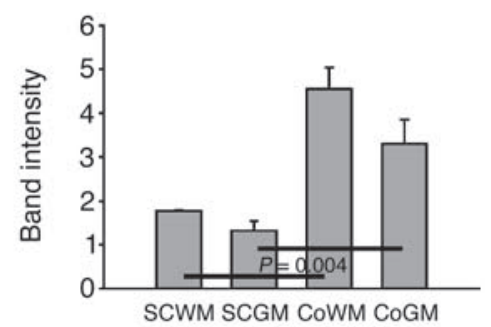

E

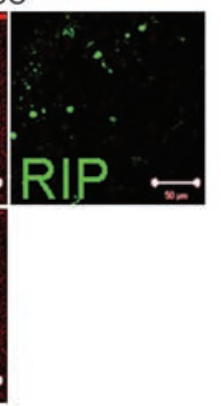

Human tissue sIL-6R

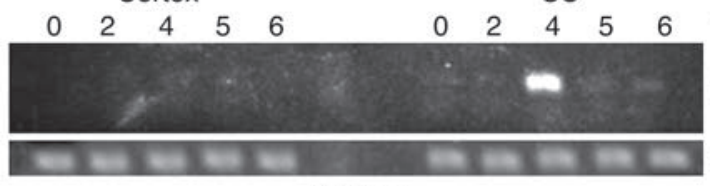

RT-PCR
H

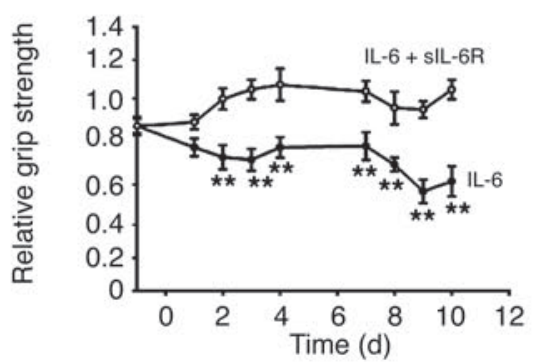

Figure 6

IL-6 induces regionally specific neural injury in the spinal cord. (A) IL-6 was administered to cortical, hippocampal, and spinal cord organotypic cultures at increasing doses, and cell death was assessed 36 hours later. Data is plotted as the fold induction of death relative to cultures with no IL-6 addition. (B) Adult rats were infused with IL-6 or saline through an intracerebroventricular (IC) cannula at the same rate (0.5 $\mu / / \mathrm{h}$ for 7 days) and concentration $(2,000 \mathrm{pg} / \mathrm{ml})$ as that previously administered via a spinal subarachnoid catheter and assessed for weakness. (C) Cortical organotypic cultures were treated with IL-6 and assessed for PARP activity up to 20 hours later. (D) Confocal microscopy of cortex and spinal cord organotypic cultures was performed after the administration of IL-6 to the culture. Scale bars: $50 \mu \mathrm{m}$. (E) RT-PCR analysis of iNOS from cortex or spinal cord organotypic cultures was performed at various times after the addition of IL-6. GAPDH serves as a PCR control. (F) Quantitative immunoblot of IL-6R expression from human autopsy tissue lysates. Spinal cord grey and white matter (SCGM and SCWM, respectively) and cortex grey and white matter (CoWM and CoGM, respectively) lysates were generated, subjected to SDS-PAGE, and probed for IL-6R immunoreactivity. (G) Quantitative immunoblot of sIL-6R from the same lysates (shown in chemiluminescent units). (H) Adult rats were infused with either IL-6 or IL-6 plus sIL-6R at a 1:1 molar ratio through a spinal subarachnoid catheter as before. Animals were assessed for hind limb grip strength for the 10-day duration of the experiment. ${ }^{*} P<0.05 ;{ }^{* \star} P<0.04$.

concentrations of IL- 6 and plotted fold induction of death relative to sections with no IL-6 administration (Figure 6A). Within hippocampal and cortical organotypic cultures, low levels of IL-6 were protective against baseline cellular injury that occurs in these cultures, while higher doses were only slightly injurious. This contrasts starkly with spinal cord organotypic cultures, which showed an injurious response to even $50 \mathrm{pg} / \mathrm{ml} \mathrm{IL-} 6$ and greater than 10 -fold increase in cell death at all greater doses tested. In adult rats, infusion of IL-6 (at the same dose previously infused into the spinal subarachnoid space) into the cerebral ventricles did not result in weakness (Figure 6B) or behavioral changes (Supplemental Figure 2), further supporting a regionally specific nervous system response to IL-6. We then repeated in cortical tissues a series of studies previously performed in the spinal cord in order to determine what limits IL-6-mediated injury in cortex organotypic cultures. We found a small early peak in PARP activity within IL-6treated cortical cultures, but did not see the second peak seen in spinal cord cultures (Figure 6C). Working further upstream, there was no accumulation of NT within cortical organotypic cultures treated with IL-6, while IL-6-treated spinal cord organotypic cultures did accumulate NT (Figure 6D), and we did not see iNOS mRNA induction (Figure 6E). JAK2 and STAT3 phosphorylation could not be detected in IL-6-treated cortical organotypic cultures (data not shown), suggesting that the regional specificity to IL-6 is even upstream of JAK and STAT. To determine the discrepancy between brain and spinal cord, we examined the expression 
of the membrane-bound IL-6R and the soluble IL-6R (sIL-6R) within human autopsy material. We found that IL-6R is similarly expressed within the brain and spinal cord, indicating that IL-6R density does not account for relative susceptibility of spinal cord tissue. There is markedly increased density of IL-6R within white matter compared to grey matter (Figure 6F), suggesting that the relative susceptibility of axons and oligodendrocytes may be mediated by this gradient. We then examined the expression of sIL-6R and found significantly greater expression in cortical tissue lysates than in spinal cord tissue lysates (Figure 6G). If sIL-6R deficiency in the spinal cord mediates the enhanced susceptibility of the spinal cord to IL-6, then co-infusion of IL- 6 and sIL-6R should blunt the effects of IL- 6 in spinal cord cellular injury. Indeed, we found that to be true, since adult rats infused with both IL- 6 and sIL-6R did not get weak, while IL- $6-$ infused animals did $(P<0.04$ at days 2-10; Figure $6 \mathrm{H})$. We suggest, therefore, that IL-6-mediated signaling is decreased in the brain relative to the spinal cord and that this impairment must be upstream of IL-6R. The observation of higher sIL-6R levels in the context of decreased IL-6 responsiveness in cortical lysates suggests that this molecule may act as an antagonist in this setting (27). Therefore, we propose that sIL-6R is a critical molecule that dampens the tissue response to IL-6. We conclude that spatially restricted responses to cytokines including IL-6 may underlie the restricted inflammation seen in a variety of CNS inflammatory disorders, including regionally limited forms of MS, TM, neuromyelitis optica, and optic neuritis.

\section{Discussion}

We hypothesized that cytokines play an important role in the pathogenesis of TM and examined the diffusible derangements within the CSF of a group of TM patients with a cytokine antibody array. We found a virtually identical pattern in the CSF from all the TM patients examined in this manner, that is, dramatic elevations in IL-6 levels. This uniformity in the pattern was surprising in that TM has widely been considered to be a heterogeneous disorder, and one may have expected the cytokine derangements to reflect this heterogeneity. However, it should be noted that recent nosologic strategies have attempted to categorize TM patients into various classifications, including monophasic vs. recurrent and idiopathic vs. those associated with systemic disease (28). For this study, we have limited the analysis to patients with idiopathic TM and have excluded those with identified systemic inflammatory disease. Therefore, this classification scheme may have resulted in a more uniform patient population with relatively homogenous immune system derangements.

Though CSF IL-6 levels reported here are among the highest reported in any human disease (up to $4,209 \mathrm{pg} / \mathrm{ml}$ ), related disorders have also been found to have elevated IL- 6 within the CNS. Acute disseminated encephalomyelitis, like TM, is a monophasic, inflammatory disorder of the CNS that is often post-infectious (29). Similarly, several reports have suggested that IL-6 is involved in the pathogenesis of MS, since patients have elevated IL- 6 in the CSF (30) and within plaques $(31,32)$ and elevated numbers of IL-6expressing monocytes within the blood and CSF (30).

There has been recent awareness about the dual role of IL- 6 as both protective and injurious (2). In contrast to the view that IL-6 may be purely injurious to the nervous system, several studies have shown that members of the IL- 6 superfamily, including IL- 6 itself, may be neuroprotective (3). These disparate effects may be explained by differential involvement of the gp130 receptor among different members of the family $(3,33)$, by distinct inflammatory pathways activated in different injury models $(7,34)$, or by a dose effect wherein low doses of IL- 6 or a family member are protective (35) while higher doses are injurious.

We found in pathological spinal cord specimens from patients with TM that astrocytes were the predominant source of IL-6 production. Astrocytes have been shown to produce IL- 6 in response to direct stimulation by proinflammatory cytokines (e.g., TNF- $\alpha$ and IL-1 $\beta$ ), viral and bacterial pathogens, and neurotransmitters. What triggers the initial biosynthesis of IL- 6 in astrocytes is currently being investigated, but potential candidates include an immune response following vaccination or an antecedent infection that could involve mechanisms such as molecular mimicry or superantigen-mediated inflammation (36). Why some individuals mount a dramatic elevation of their IL- 6 levels that results in the pathophysiological injury seen in TM is still unknown, but the potential contribution of genetic differences to CNS IL- 6 production has been previously described $(37,38)$.

Primary targets of IL-6-mediated cytotoxicity include oligodendrocytes and axons. The finding of NT accumulation in axons argues for a direct neuropathic effect of IL- 6 in mediating neural injury, as opposed to axonal degeneration that is solely the result of demyelination. Our finding that APP axonal transport was blocked in the spinal cords of TM patients is consistent with a recent report that microglial iNOS activation produces a breakdown in axonal transport (39). Both demyelination and axonal degeneration are hallmarks of CNS autoimmune demyelinating conditions, such as TM and MS, and these pathological hallmarks were reproduced in adult rats in which IL-6 was infused into their spinal cords.

Excessive NO production can lead to cellular injury by several mechanisms, including loss of membrane integrity through direct lipid peroxidation, altered protein function through NT formation, or energy depletion through PARP activation (40). Studies with PARP-1 KO mice and chemical PARP inhibitors have consistently shown that lack of PARP activation following severe oxidative injury with $\mathrm{NO}$, hydrogen peroxide, and $\mathrm{ONOO}^{-}$results in significantly reduced neuronal death (22). It was recently shown that $\mathrm{ONOO}^{-}$exerts toxic effects on spinal cord neurons at least in part through a PARP-dependent pathway, as demonstrated by the ability of PARP inhibitors to preserve neuronal viability in the presence of $\mathrm{ONOO}^{-}(20)$.

Using spinal cord organotypic cultures, we observed a biphasic activation of PARP: the first peak, occurring 2 hours after addition of IL-6, was not dependent upon iNOS, while the later peak was. This suggests that NO production was necessary for the second peak of PARP activity, presumably by initiating DNA damage that resulted in increased PARP activation. The observation that PARP inhibition prevented IL-6-mediated spinal cord cell death both in organotypic cultures and in the adult rat spinal cord supports the importance of PARP as a final effector in the pathway of IL-6mediated cellular injury in vivo.

The first peak of PARP activity after IL- 6 administration coincides with STAT phosphorylation and not NO production. Since IL-6 is capable of mediating JAK/STAT signaling pathways other than JAK2/STAT3 and Ras/MEK/MAPK (11), we speculate that PARP has a role in signal transduction. Recent evidence from PARP KO mice suggests a role for PARP in enhancing p38/MAPK signaling in response to inflammatory stimulation through lipopolysaccharide (LPS) (41). Perhaps the early-phase IL-6-mediated PARP activation we describe here plays a role in 
the augmentation of p38/MAPK signaling. Spinal cord iNOS activation, however, is not due to early PARP activation, because it is unaffected by PARP inhibition.

Previous studies have implicated IL-6 family members in preventing cell death as well as potentially playing a causative role in neurodegenerative diseases (2). This discrepancy may result from differences in downstream signaling among IL- 6 and its family members and differing effects in distinct experimental paradigms. The protective or destructive actions of IL- 6 may also result from selective dose and regional effects. We observed that IL- 6 causes preferential cytotoxicity in white matter compared to gray matter in the spinal cord. We also found that low doses of IL- 6 prevented cell death in organotypic cultures of sections from the hippocampus or cortex, whereas higher doses had little effect on cell death. In contrast, no IL-6 dose tested in spinal cord sections was found to be protective, and higher doses were extremely cytotoxic. The observation that IL-6 treatment of cortical organotypic cultures did not cause significant PARP activation, iNOS induction, or STAT phosphorylation suggests that the regional selectivity of IL- 6 response occurs at the level of IL-6R and not its downstream signaling pathway.

The increased abundance of IL-6R in white compared to grey matter, which may be due to increased abundance of IL-6R on oligodendrocytes, may account for the preferential white matter injury seen both in vitro and in vivo following IL-6 application. However, it is the higher concentration of sIL-6R that may explain the increased susceptibility of the spinal cord to IL-6. Alternative explanations for the role of sIL-6R in preventing cell death include the possibilities that IL- 6 is bound and isolated by sIL-6R or that the IL-6/sIL-6R complex initiates signaling through alternative pathways in cells without IL-6R (42). Biological markers of regional selectivity within the CNS has been seen previously, such as the selective clearance of alphavirus from spinal cord relative to the cortex (43) and the selective vulnerability of spinal cord neurons to excitotoxic injury when compared to cortical neurons.

We have found in this study that a single signaling molecule is a critical determinant of patient outcome in TM. The implications of these findings are that therapeutic strategies capable of modulating this pathway may improve outcomes in TM patients. Although IL-6, iNOS, and PARP have each been indirectly implicated in the pathophysiology of neuroinflammatory disorders previously (2, $12,22)$, our work provides direct evidence for a signaling cascade involving these proteins that accounts for the clinicopathologic findings in inflammatory spinal neurodegeneration. Since spinal cord dysfunction is a major determinant of disability in several neurologic disorders including TM and MS, the elucidation of this pathway identifies important therapeutic targets for preventing this disability in the future.

\section{Methods}

Study subjects. All patients underwent a lumbar puncture at acute onset of disease as part of their clinical care and diagnosis prior to initiating treatment and consented to the withdrawal of additional CSF for research (approved by the Johns Hopkins Medicine Institutional Review Board). Idiopathic TM patients were defined according to previously published criteria (1). Those who did not meet the inflammatory criteria were classified as noninflammatory controls. Spinal fluid was collected, immediately placed on ice, centrifuged at $1,000 \mathrm{~g}$ to remove cellular elements, and stored at $-80^{\circ} \mathrm{C}$.

Clinical assessments. Clinical and radiological data was collected: the presence of gadolinium enhancement; the location and extent of the lesion on the spinal MRI; and the levels of protein and number of white blood cells, if any, in the CSF during the acute phase (see Supplemental Table 1). Further, functional status (acute and follow-up EDSS scores) were assessed by neurologists who were blinded to the immunologic assay results. The EDSS is a widely used neurological rating scale, ranging from 0 (normal) to 10 (death).

Immunologic assays. Cytokine antibody arrays were purchased (TranSignal RayBio Human Cytokine Antibody Array 3, catalog no. MA6020; Panomics Inc.) and used according to package inserts. For each blot, 1,000 $\mu$ l of CSF was used. Signal was analyzed and quantitated by using a Fuji chemiluminescent detection system. Quantitative IL-6 ELISA assay kits and total NO assay kits were purchased from R\&D Systems and the LIVE/DEAD Viability/Cytotoxicity Kit was purchased from Invitrogen Corp.; all were used according to the manufacturers' instructions. All samples were measured in triplicate and average values were determined. Total nitrite concentrations in supernatants collected from in vitro coculture experiments were determined by using the Total NO Kit (catalog no. DE1600; R\&D Systems) as specified by the manufacturer. PARP activity was assayed using a PARP Activity Assay Kit (catalog no. 4667-50-K; Trevigen) according to the manufacturer's instructions. NT release in supernatants collected from in vitro coculture experiments was analyzed using the Nitrotyrosine ELISA Test Kit (catalog no. HK501; Cell Sciences) as directed by the manufacturer.

Reagents. Recombinant rat IL-6 (catalog no. 557008; BD Biosciences - Pharmingen) was made as a $2 \mu \mathrm{g} / \mathrm{ml}$ stock in $1 \mathrm{mg} / \mathrm{ml} \mathrm{BSA}$ in PBS and used at final concentrations of $500 \mathrm{pg} / \mathrm{ml}$ and 2,000 pg/ml. iNOS inhibitor 1400W dihydrochloride (catalog no. ALX-270-073; Alexis Biochemicals) was made at $100 \mathrm{mM}$ stock in water and used at a final concentration of $100 \mu \mathrm{M}$. We also dissolved 4-ANI (catalog no. ALX-270-250-M010; Alexis Biochemicals) in $10 \%$ DMSO and used this for in vitro studies at a final concentration of $5 \mu \mathrm{M}$ from a 5.8-mM stock. We obtained 3-AB from Sigma-Aldrich (catalog no. A0788). Microglial identity was determined by incubation with DiI-Ac-LDL (Invitrogen Corp.). This marker identifies phagocytosing cells and has been reported to accurately identify microglial cells in the nervous system (44).

Antibodies and dilutions used in this study include the following: PhosphoPlus Stat3 (Tyr705) Antibody Kit (1:1,000; Cell Signaling Technology); iNOS/NOS Type II (1:10,000; BD Biosciences); anti-NT, clone 1A6 (1:1,000; Upstate); PARP antibody (1:75; Cell Signaling Technology), biotinylated anti-human IL-6R antibody (1:2,500; R\&D Systems), RIP (SMI 91; Sternberger Monoclonals), neurofilament (NF), Heavy Chain (SMI 31/32; Sternberger Monoclonals), and GFAP (1:400, catalog no. MAB360, or 1:2,000, catalog no. AB5804; Chemicon). CSF samples from TM patients were immunodepleted with anti-human IL-6 antibodies (catalog no. ab6672; Novus Biologicals), precipitated with protein A-coated beads, and verified for IL-6 depletion by human IL-6 ELISA assay.

Immunoblots. Western blots were developed with SuperSignal West Femto Maximum Sensitivity Substrate (Pierce Biotechnology Inc.) and quantified with a Luminescent Image Analyzer (LAS-1000 plus camera) and Image Gauge software (Fuji). Protein expression in treated tissue was normalized to levels in untreated controls by dividing measured band intensities. Supernatants were collected at each time point and centrifuged at 13.2 $\mathrm{rpm}$ in a tabletop centrifuge for 5 minutes at $4^{\circ} \mathrm{C}$. The clarified supernatant was then analyzed for the presence of total nitrites and NT.

Rat spinal cord organotypic cultures. All animal experiments were approved by the Johns Hopkins Animal Care and Use Committee, and mice were cared for and euthanized according to the guidelines of the committee. Spinal cord organotypic slice cultures were prepared from 8-day-old Sprague-Dawley rats as previously described (45). Growth media was changed 1 day after culturing and twice more before using. Cultures were used 7-10 days after culturing. Cultures were changed to serum-free organotypic culture media and treated with recombinant human IL-6, PBS, or $100 \mu \mathrm{l}$ human CSF from TM patients. 
Microglial cultures. Microglial cultures were isolated from adult rats as described by Babas et al. (44), with a few modifications. The cells were derived from the cortical regions of 3 adult Lewis rat brains. Processed tissues were passed through an 18-gauge needle on a 10-CC syringe and filtered over 70- $\mu \mathrm{m}$ nylon cell strainers (Falcon, catalog no. 352350; BD Biosciences) twice to ensure a single cell suspension. Cells were plated at a density of $4 \times 10^{6}$ cells/well. The next day, cells were washed and changed to fresh growth medium. Cells were maintained for a week before experimentation. Microglial cell purity (at least 90\%) was verified by fluorescent staining of microglia with Iba-1 (1:150, catalog no. 01-1974; Wako) and the absence of staining with GFAP.

Spinal cannulated rats. Adult Sprague-Dawley rats with spinal cannulas were purchased from Zivic Laboratories Inc. These rats had cannulas placed into the cisterna magna and extended caudally through the subarachnoid space with the cannula tip terminating adjacent to the T8 vertebral body. Cannulated rats were anesthetized with avertin, an incision was made behind the head, and the subarachnoid cannula was connected to an Alzet pump (1007D, $0.5 \mu \mathrm{l} / \mathrm{hr}$ for 7 days). Pumps were filled with $100 \mu \mathrm{lL}-6$ at $2 \mu \mathrm{g} / \mathrm{ml}$ or saline. Animals were coded and housed individually. There were 10 rats per group, and each rat was scored for hind limb grip strength daily by a blinded examiner. Animals were perfused with $4 \%$ paraformaldehyde or $2 \%$ paraformaldehyde $/ 2 \%$ glutaraldehyde 7 days after initiating IL- 6 infusion, and spinal cords were harvested for immunohistochemistry or plastic sectioning. Plastic sections $(1 \mu \mathrm{M})$ were generated of the lumbar spinal cord after osmium tetroxide embedding. Additional behavioral studies were carried out as described in Supplemental Methods.

Statistics. SPSS software (version 12.0; SPSS Inc.) was used for all statisti$\mathrm{cal}$ analyses. Box plots were used to represent the distribution of the data. The outliers shown are outside the fifth and ninety-fifth percentile. Correlations were assessed by Spearman's rank correlation coefficient due to the ordinal nature of the data. Group differences in EDSS and IL-6 levels and between CSF and serum IL-6 levels in the different patient groups were compared using the Mann-Whitney $U$ test due to the non-Gaussian appearance of the data. A $P$ value less than 0.05 was considered significant. Due to the nonparametric nature of the data (as determined by using tests of normality), nonparametric equivalent tests of ANOVA and repeated-measures ANOVA were used to increase the robustness of the results. The Kruskal-Wallis test was performed to analyze differences among groups at each time point, and Friedman's nonparametric repeated measures comparison was used to analyze differences across time within a group. The MannWhitney $U$ test was used for the comparison of 2 independent samples.

\section{Acknowledgments}

We thank Lawrence S. Mayer, Solomon Snyder, and Timothy Moran for their critiques of this manuscript. We recognize the support of the Transverse Myelitis Association, the Noel P. Rahn Fellowship, the Dana Foundation, the Miriam and Peter Haas Foundation, the Katie Sandler Fund for Research at Johns Hopkins University, Bruce Downey, and Barr Laboratories. We also acknowledge the support of the General Clinical Research Center (GCRC) of the Johns Hopkins School of Medicine (NIH/National Center for Research Resources grant M01-RR000052). C.A. Pardo's work is supported by a NIH/National Institute on Drug Abuse K08 award (DA16160).

Received for publication March 25, 2005, and accepted in revised form July 19, 2005

Address correspondence to: Douglas A. Kerr, Department of Neurology, Johns Hopkins University School of Medicine, Pathology 627 C, 600 North Wolfe Street, Baltimore, Maryland 21287, USA. Phone: (410) 955-3129; Fax: (410) 502-6736; E-mail: dkerr@jhmi.edu.
1. Krishnan, C., Kaplin, A.I., Deshpande, D.M., Pardo, C.A., and Kerr, D.A. 2004. Transverse myelitis: pathogenesis, diagnosis and treatment. Front. Biosci. 9:1483-1499.

2. Gadient, R.A., and Otten, U.H. 1997. Interleukin-6 (IL-6)--a molecule with both beneficial and destructive potentials. Prog. Neurobiol. 52:379-390.

3. Ransohoff, R.M., Howe, C.L., and Rodriguez, M. 2002. Growth factor treatment of demyelinating disease: at last, a leap into the light. Trends Immunol. 23:512-516.

4. Gruol, D.L., and Nelson, T.E. 1997. Physiological and pathological roles of interleukin- 6 in the central nervous system. Mol. Neurobiol. 15:307-339.

5. Wilson, C.J., Finch, C.E., and Cohen, H.J. 2002 Cytokines and cognition--the case for a head-totoe inflammatory paradigm. J. Am. Geriatr. Soc. 50:2041-2056.

6. Campbell, I.L., et al. 1993. Neurologic disease induced in transgenic mice by cerebral overexpression of interleukin 6. Proc. Natl. Acad. Sci. U. S. A. 90:10061-10065.

7. Okuda, Y., et al. 1998. IL-6-deficient mice are resistant to the induction of experimental autoimmune encephalomyelitis provoked by myelin oligodendrocyte glycoprotein. Int. Immunol. 10:703-708.

8. Gijbels, K., Brocke, S., Abrams, J.S., and Steinman, L. 1995. Administration of neutralizing antibodies to interleukin-6 (IL-6) reduces experimental autoimmune encephalomyelitis and is associated with elevated levels of IL- 6 bioactivity in central nervous system and circulation. Mol. Med. 1:795-805.

9. Tuna, M., et al. 2001. Effect of anti-rat interleukin- 6 antibody after spinal cord injury in the rat: inducible nitric oxide synthase expression, sodium- and potassium-activated, magnesium-dependent adenosine-5'-triphosphatase and superoxide dismutase activation, and ultrastructural changes. J. Neurosurg. 95:64-73.

10. Yu, X., Kennedy, R.H., and Liu, S.J. 2003. JAK2/ STAT3, not ERK1/2, mediates interleukin-6induced activation of inducible nitric-oxide synthase and decrease in contractility of adult ventricular myocytes. J. Biol. Chem. 278:16304-16309.

11. Van Wagoner, N.J., and Benveniste, E.N. 1999. Interleukin- 6 expression and regulation in astrocytes. J. Neuroimmunol. 100:124-139.

12. Liberatore, G.T., et al. 1999. Inducible nitric oxide synthase stimulates dopaminergic neurodegeneration in the MPTP model of Parkinson disease. Nat. Med. 5:1403-1409.

13. Abramson, S.B., Amin, A.R., Clancy, R.M., and Attur, M. 2001. The role of nitric oxide in tissue destruction. Best Pract. Res. Clin. Rheumatol. 15:831-845.

14. Dawson, V.L., Brahmbhatt, H.P., Mong, J.A., and Dawson, T.M. 1994. Expression of inducible nitric oxide synthase causes delayed neurotoxicity in primary mixed neuronal-glial cortical cultures. Neuropharmacology. 33:1425-1430.

15. Lieb, K., Engels, S., and Fiebich, B.L. 2003. Inhibition of LPS-induced iNOS and NO synthesis in primary rat microglial cells. Neurochem. Int. 42:131-137.

16. Calabrese, V., et al. 2002. Nitric oxide synthase is present in the cerebrospinal fluid of patients with active multiple sclerosis and is associated with increases in cerebrospinal fluid protein nitrotyrosine and S-nitrosothiols and with changes in glutathione levels. J. Neurosci. Res. 70:580-587.

17. Shin, T., Kim, S., Moon, C., Wie, M., and Kim, H. 2000. Aminoguanidine-induced amelioration of autoimmune encephalomyelitis is mediated by reduced expression of inducible nitric oxide synthase in the spinal cord. Immunol. Invest. 29:233-241.

18. Isaksson, J., Farooque, M., and Olsson, Y. 2005. Improved functional outcome after spinal cord injury in iNOS-deficient mice. Spinal Cord. 43:167-170.

19. Blantz, R.C., and Munger, K. 2002. Role of nitric oxide in inflammatory conditions. Nephron. 90:373-378.

20. Scott, G.S., Szabo, C., and Hooper, D.C. 2004. Poly(ADP-ribose) polymerase activity contributes to peroxynitrite-induced spinal cord neuronal cell death in vitro. J. Neurotrauma. 21:1255-1263.

21. Burkle, A. 2001. Physiology and pathophysiology of poly(ADP-ribosyl)ation. Bioessays. 23:795-806.

22. Ha, H.C., and Snyder, S.H. 2000. Poly(ADP-ribose) polymerase- 1 in the nervous system. Neurobiol. Dis. 7:225-239.

23. Genovese, T., et al. 2005. Inhibitors of poly (ADPribose) polymerase modulate signal transduction pathways and secondary damage in experimental spinal cord trauma. J. Pharmacol. Exp. Ther. 312:449-457.

24. Irani, D.N., and Kerr, D.A. 2000. 14-3-3 protein in the cerebrospinal fluid of patients with acute transverse myelitis [letter]. Lancet. 355:901.

25. Radi, R. 2004. Nitric oxide, oxidants, and protein tyrosine nitration. Proc. Natl. Acad. Sci. U. S. A. 101:4003-4008.

26. Hill, K.E., Zollinger, L.V., Watt, H.E., Carlson, N.G., and Rose, J.W. 2004. Inducible nitric oxide synthase in chronic active multiple sclerosis plaques: distribution, cellular expression and association with myelin damage. J. Neuroimmunol. 151:171-179.

27. Michalopoulou, M., et al. 2004. Soluble interleu- 
kin-6 receptor (sIL-6R) in cerebrospinal fluid of patients with inflammatory and non inflammatory neurological diseases. Immunol. Lett. 94:183-189.

28. Transverse Myelitis Consortium Working Group. 2002. Proposed diagnostic criteria and nosology of acute transverse myelitis [review]. Neurology. 59:499-505.

29. Ravin, P., and Hedley-Whyte, E.T. 2002. Case records of the Massachusetts Genera Hospital. Weekly clinicopathological exercises. Case 34-2002. A 55-year-old man with cognitive and sensorimotor findings and intracranial lesions. N. Engl. J. Med. 347:1433-1440.

30. Miljkovic, D.J., et al. 2002. Nitric oxide metabolites and interleukin- 6 in cerebrospinal fluid from multiple sclerosis patients. Eur. J. Neurol. 9:413-418.

31. Mycko, M.P., Papoian, R., Boschert, U., Raine, C.S., and Selmaj, K.W. 2003. cDNA microarray analysis in multiple sclerosis lesions: detection of genes associated with disease activity. Brain. 126:1048-1057.

32. Lock, C., et al. 2002. Gene-microarray analysis of multiple sclerosis lesions yields new targets validated in autoimmune encephalomyelitis. Nat. Med.
8:500-508.

33. Ishihara, K., and Hirano, T. 2002. Molecular basis of the cell specificity of cytokine action. Biochim. Biophys. Acta. 1592:281-296.

34. Rodriguez, M., Pavelko, K.D., McKinney, C.W., and Leibowitz, J.L. 1994. Recombinant human IL-6 suppresses demyelination in a viral model of multiple sclerosis. J. Immunol. 153:3811-3821.

35. Zhang, P., Chebath, J., Lonai, P., and Revel, M. 2004 Enhancement of oligodendrocyte differentiation from murine embryonic stem cells by an activator of gp130 signaling. Stem Cells. 22:344-354

36. Kaplin, A.I., Krishnan, C., Deshpande, D.M., Pardo, C.A., and Kerr, D.A. 2005. Diagnosis and management of acute myelopathies. Neurologist. 11:2-18.

37. Lynch, J.R., et al. 2003. APOE genotype and an ApoE-mimetic peptide modify the systemic and central nervous system inflammatory response. J. Biol. Chem. 278:48529-48533.

38. Takeda, K., et al. 1999. Enhanced Th1 activity and development of chronic enterocolitis in mice devoid of Stat 3 in macrophages and neutrophils. Immunity. 10:39-49.
39. Stagi, M., et al. 2005. Breakdown of axonal synaptic vesicle precursor transport by microglial nitric oxide. J. Neurosci. 25:352-362.

40. Kiefmann, R., et al. 2004. Role of PARP on iNOS pathway during endotoxin-induced acute lung injury. Intensive Care Med. 30:1421-1431.

41. Ha, H.C. 2004. Defective transcription factor activation for proinflammatory gene expression in poly(ADP-ribose) polymerase 1-deficient glia. Proc. Natl. Acad. Sci. U. S. A. 101:5087-5092.

42. Regan, R.F. 1996. The vulnerability of spinal cord neurons to excitotoxic injury: comparison with cortical neurons. Neurosci. Lett. 213:9-12.

43. Binder, G.K., and Griffin, D.E. 2001. Interferongamma-mediated site-specific clearance of alphavirus from CNS neurons. Science. 293:303-306.

44. Babas, T., et al. 2003. Role of microglial cells in selective replication of simian immunodeficiency virus genotypes in the brain. J. Virol. 77:208-216.

45. Rothstein, J.D., Jin, L., Dykes-Hoberg, M., and Kuncl, R.W. 1993. Chronic inhibition of glutamate uptake produces a model of slow neurotoxicity. Proc. Natl. Acad. Sci. U. S. A. 90:6591-6595. 